\title{
Efficacy and safety of HCV-treatment with direct-acting antiviral agents interferon-free, in patients with severe renal impairment in clinical practice
}

\author{
J. Cabezas ${ }^{1,2}$, M.C. Londoño ${ }^{3}$, S. Llerena ${ }^{1,2}$, R. Muñoz ${ }^{4}$ C. Baliellas ${ }^{5}$, M. Buti ${ }^{6}$, J.M. \\ Pascasio $^{7}$, F. Sáez-Royuela ${ }^{8}$, J. García-Samaniego ${ }^{9}$, J. de la Vega ${ }^{10}$, P. Sánchez-Pobre ${ }^{11}$, C. \\ Álvarez-Navascues ${ }^{12}$, J.A. Carrión ${ }^{13}$, F. Gea ${ }^{14}$, R.M. Morillas ${ }^{15}$, M. Fernández-Bermejo ${ }^{16}$, A. \\ Castro $^{17}$, M. Rivero ${ }^{18}$, J.R. Fernández ${ }^{19}$, M. Berenguer ${ }^{20}$, J.J. Moreno ${ }^{21}$,D. Rincón ${ }^{22}$, J.L. \\ Calleja $^{23}$, J. Crespo ${ }^{1,2}$.
}

\begin{abstract}
${ }^{1}$ Digestive Service, Hospital Universitario Marqués de Valdecilla ${ }^{2}$ Instituto Investigación Valdecilla, IDIVAL, Santander; ${ }^{3}$ Hospital Clinic, Barcelona; ${ }^{4}$ Hospital Doce de Octubre, Madrid; ${ }^{5}$ Hospital Bellvitge, Hospitalet; ${ }^{6}$ Hospital Vall d'Hebron, Barcelona; ${ }^{7}$ Hospital Universitario Virgen del Rocio, Sevilla; ${ }^{8}$ Hospital Universitario de Burgos, Burgos; ${ }^{9}$ Hospital Universitario La Paz, Madrid; ${ }^{10}$ Hospital San Agustin, Aviles; ${ }^{11}$ Hospital Universitario Clinico San Carlos, Madrid; ${ }^{12}$ Hospital Central de Asturias, Oviedo; ${ }^{13}$ Hospital del Mar, Barcelona; ${ }^{14}$ Hospital Ramon y Cajal, Madrid; ${ }^{5}$ Hospital Germans Trias i Pujol, Badalona; ${ }^{16}$ Hospital San Pedro de Alcantara, Caceres; ${ }^{17}$ Hospital Universitario A Coruña, A Coruña; ${ }^{18}$ Hospital Sureste, Almeria; ${ }^{19}$ Hospital Universitario de Cruces, Bilbao; ${ }^{20}$ Hospital La Fe, Valencia $;{ }^{21}$ Hospital General Segovia, Segovia; ${ }^{22}$ Hospital Universitario Gregorio Marañon, ${ }^{23}$ Hospital Universitario Puerta de Hierro, Madrid, Spain
\end{abstract}

Background and Aims: Chronic hepatitis $\mathrm{C}$ virus infection (CHC) increases the risk of death in patients with chronic kidney disease (CKD) stage $4-5$. However, patients with hepatitis $\mathrm{C}$ and $\mathrm{CKD}$ are considered a special population who are difficult to treat, and information about the efficacy and safety of IFN -free treatment regimens is limited. The aim of this study is to analyze the effectiveness and safety of the free IFN therapy in a large number of patients with CHC and CKD in clinical practice in Spain.

Methods: We carried out an observational, ambispective, multicenter study that included 1,343 Hep-C patients from the Spanish Association for the Study of the Liver (AEEH) database. Among those patients, 100 have advanced CKD data: 45 patients are stage $3 \mathrm{~b}(\mathrm{Cl}$ cret $30-44 \mathrm{~mL} / \mathrm{min}), 18$ are stage $4(\mathrm{Cl}$ cret $15-30 \mathrm{~mL} / \mathrm{min})$ and 37 are stage $5(\mathrm{Cl}$ cret $<15 \mathrm{~mL} / \mathrm{min}$, with/without hemodialysis $)$. All patients began treatment before October 2015. Demographic, clinical, virological, pretreatment type, antiviral regimen and renal function variables were analyzed.

Results: Patients with CKD were mostly men (74\%) with a mean age of 57.6 years. Of the 100 patients, only 33 $(33 \%)$ had undergone a previous antiviral therapy, compared with $760(61.3 \%)$ patients without CKD (p < 0.001). Genotype distribution: G1 (86\%); G3 (5\%); G4 (9\%). Fibrosis distribution: F0-F1: 22/F2-F3: 28/F4: 50. CKD patients were treated with SOF + SIM + RBV (8), SOF + LDV + RBV (16), SOF + DCV + RBV (16;), SIM + DCV + RBV (48), 3D Abbvie + RBV (9) SOF + RBV (3). The SVR4 (CKD 35/39; 89.7\%) and SVR12 $(42 / 47 ; 89.3 \%)$ were similar to patients without CKD (405/449; 90.2\%). Dependant on the type of treatment, the SVR12 was: SOF + SIM + - RBV (7/8; 87.5\%), SOF + LDV + / - RBV (2/2, 100\%), SOF + DCV +/ - RBV $(16 / 16 ; 100 \%)$, SIM + DCV +/ - RBV (14/17; 82.3\%), 3D Abbvie (1/1; 100\%) SOF + RBV (2/3; 66,6\%). SVR was lower in patients with cirrhosis $(19 / 22,86.3 \%)$ than in the rest of the cohort $(18 / 18 ; 100 \% ; \mathrm{p}=0.024)$. The stage of CKD did not influence the possibility of obtaining an SVR. Similarly, the SVR was independent of the CV [N1], genotype, duration of therapy, and whether or not RBV was used. No significant safety problems occurred, however treatment was discontinued in two patients due to a progression in renal impairment.

Conclusions: This multicenter clinical practice study demonstrates the remarkable effectiveness and safety of the combination of different IFN-free regimens in patients with $\mathrm{CHC}$ and CKD. 